\title{
ESTUDO DA INTER-RELAÇÃO DA QUALIDADE DO SOLO E DA SERAPILHEIRA COM A FAUNA EDÁFICA UTILIZANDO ANÁLISE MULTIVARIADA
}

MANHAES, Carmen Maria Coimbra ${ }^{1}$

FRANCELINO, Francisco Maurício Alves ${ }^{2}$

RESUMO: O solo é o substrato natural para grande parte da biodiversidade global e está entre os mais biologicamente ricos em habitats e espécies do planeta. Os organismos do solo exercem inúmeras funções essenciais para o biofuncionamento dos solos e por esta razão, torna-se importante caracterizá-los e conhecer quais são os fatores que mais afetam a distribuição das comunidades que habitam o sistema solo-serapilheira. Os organismos edáficos têm papel importante na identificação da qualidade do solo, por atuarem nos processos de transformação da matéria orgânica, ciclagem de nutrientes e interações de troca com a maioria das espécies de plantas. Estes organismos são afetados por fatores como temperatura, umidade, textura, porosidade, entre outros. Muitos dos organismos do solo preferem ambientes úmidos e a presença de argila pode assegurar elevada capacidade de retenção de água no solo.

Palavras-chave: Fauna do solo. Qualidade do solo. Análise multivariada. Análise de trilha.

SUMMARY: The Soil is the natural substrate for the large part of global biodiversity and is among the most biologically rich habitats and species on the planet. Soil organisms perform numerous functions essential to the biofunctional soil and for this reason, it becomes important to characterize them and to know which are the most important factors affecting the distribution of the communities that inhabit the soil-litter system. The soil organisms play an important role in the identification of soil quality; they work on processing of organic matter, nutrient cycling and exchange interactions with most plant species. These organisms are affected by factors such as temperature, moisture, texture, porosity, among others. Many soil organisms prefer moist environments and the presence of clay can provide high capacity for water retention in soil.

Keywords: Soil fauna. Soil quality. Multivariate analysis. Path analysis.

\section{INTRODUÇÃO}

Para o produtor rural, o solo é um bem muito precioso. Porém muitos ainda não se deram conta disto e não o tratam da forma como ele deveria ser tratado. O solo deve ser tratado como um bem precioso e finito. O solo deve ser considerado como vida e sustento de vida, sustento tanto da vida humana quanto da vida dos seus habitantes, aqueles organismos vistos e não vistos a olho nu.

Estes habitantes do solo são chamados de fauna do solo. Eles exercem um precioso papel na regulação dos sistemas agrícolas e segundo Devide; Castro, (2009), desde os anos 1980, a fauna do solo vem sendo pesquisada no Brasil como indicador de sustentabilidade do manejo dos solos tropicais.

A fauna do solo tem importante atuação nos processos de decomposição, mineralização e humificação de resíduos orgânicos; imobilização e mobilização de macro e micronutrientes; fixação de

\footnotetext{
${ }^{1}$ Engenheira Agrônoma, Mestre em Produção Vegetal - Solos e Nutrição de plantas, Doutoranda em produção Vegetal - Engenharia e Economia na agricultura.

${ }^{2}$ Licenciado em Ciências Agrícolas, Mestre em Produção Vegetal - Experimentação Agropecuária.
} 
nitrogênio atmosférico; estruturação e agregação do solo e conseqüente conservação e regulação de pragas e doenças (auto-regulação), beneficiando os sistemas de produção como um todo (DEVIDE; CASTRO, 2009).

A fauna edáfica é muito sensível às ações humanas de manejo. Deste modo, Moço et al. (2005) relatam que alterações na densidade e diversidade da fauna do solo em ecossistemas ou agrossistemas que sofreram algum tipo de intervenção na sua cobertura vegetal constituem-se num bom indicador de mudanças ocorridas nesses sistemas.

Diversos estudos têm demonstrado que a mesofauna e a macrofauna são muito sensíveis ás modificações da cobertura e da estrutura do solo, se tornando um alvo fácil para identificar o processo de degradação e empobrecimento do solo. Segundo Moço (2006), os organismos edáficos são sensíveis às práticas de manejo, ao clima, às propriedades do solo e à vegetação, sendo afetados por fatores como qualidade e quantidade da matéria orgânica, $\mathrm{pH}$, temperatura, umidade, textura, porosidade e teores de nutrientes. Devido à grande influência que estas características exercem sobre a fauna edáfica, estudar a interrelação da fauna do solo com a qualidade química da serapilheira e do solo torna-se importante e necessário.

Para estudarmos estas interrelações podemos utilizar a análise de variância multivariada, pois é uma técnica de grande relevância para a derivação de inferências referentes à variação de um conjunto de variáveis-respostas entre dois ou mais tratamentos. Segundo Coimbra et al. (2007), a utilização desta técnica pode adicionar informações relevantes à variação conjunta de variáveis-respostas, e tendendo a dar uma resposta biologicamente mais coerente. Pois ela considera a dependência entre as variáveis-respostas (covariância), fato que na análise univariada não é contemplado. Métodos de análise multivariada são importantes no estudo das alterações de atributos do solo (MALUCHE-BARETTA et al., 2006).

A análise de apenas uma variável pode ser uma simplificação perigosa, não evidenciando de forma adequada as verdadeiras causas de variações intrínsecas aos dados do estudo, e deixando fora da análise a importante informação de covariabilidade entre as variáveis (COIMBRA et al., 2007). Então surge a necessidade da análise multivariada toda vez que o pesquisador tem vários atributos a serem analisados e necessita estudar simultaneamente suas relações (MARIANI et al., 2006) e estas podem ser diretas ou indiretas. Podemos avaliar as relações diretas e indiretas de um determinado atributo sobre outro utilizando a análise de trilha, desenvolvida por Wright (1923), e pormenorizada por Li (1975). A análise de trilha consiste no estudo dos efeitos diretos e indiretos de caracteres de uma variável sobre outra.

Devido à grande influência que as características químicas e físicas do solo e da serapilheira exercem sobre a fauna edáfica, estudar a interrelação da fauna do solo com estas qualidades torna-se justificável. Deste modo, o objetivo do presente trabalho é fazer uma revisão de literatura relatando a interrelação existente e comprovada por vários autores entre a qualidade do solo e da serapilheira e a fauna edáfica.

\section{REVISÃO DE LITERATURA}

\section{Fauna do solo}

A fauna do solo está intimamente ligada aos processos de decomposição e ciclagem de nutrientes, que são de capital importância para a manutenção da produtividade do ecossistema. A fauna do solo é constituída por organismos invertebrados que vivem permanentemente no solo ou que passam pelo menos um ciclo de sua vida no solo.

Nucleus, v.9, n.2, out.2012 
A fauna do solo é dividida em micro, meso e macrofauna de acordo com seu tamanho corporal. A microfauna do solo cujo diâmetro corporal varia entre $4 \mu \mathrm{m}$ e $10 \mu \mathrm{m}$ é representada em sua maioria por protozoários, rotíferos, nematóides, etc. (CORREIA; ANDRADE, 1999). Podem afetar a estrutura do solo através de interações com a microflora. Estes pequenos animais atuam de maneira indireta na ciclagem de nutrientes através da ingestão de bactérias e fungos. A intensidade de predação pode, em muitos casos, intensificar a mineralização ou retardar a imobilização de nutrientes na biomassa microbiana (CORREIA; OLIVEIRA, 2000). Os animais da microfauna geralmente vivem nos filmes de água do solo e alimentamse de microrganismos, raízes de plantas e outros organismos da microfauna ou algumas vezes de organismos maiores (BROWN et al., 2006).

Os organismos pertencentes à mesofauna estão compreendidos entre populações de $10 \mu \mathrm{m}$ a $2 \mathrm{~mm}$, a mesofauna é formada por ácaros, colêmbolos, aracnídeos, diversas ordens de insetos e alguns oligoquetos. Suas atividades tróficas incluem tanto o consumo de microrganismos e da microfauna, como também a fragmentação do material vegetal em decomposição (saprofagia), alterando a ciclagem de nutrientes, afetando a estrutura do solo produzindo pelotas fecais, criando bioporos, promovendo a humificação (CORREIA; OLIVEIRA, 2000). Possuem habilidade de escavar, geralmente vivem nos poros do solo e podem também alimentar-se de matéria orgânica (BROWN et al., 2006).

Já a macrofauna apresenta diâmetro corporal entre $2 \mathrm{~mm}$ e $20 \mathrm{~mm}$ e podem pertencer a quase todas as ordens encontradas na mesofauna, excetuando-se ácaros, colêmbolos, proturos e dipluros. Regulam as populações de fungos e da microfauna, estimulam a atividade microbiana, podendo afetar a estrutura do solo, misturando partículas orgânicas e minerais, redistribuindo a matéria orgânica e microrganismos, promovendo a humificação e produzindo pelotas fecais. (CORREIA; OLIVEIRA, 2000).

A habilidade dos animais do solo em transportar, ingerir, ou modificar a estrutura física do solo é geralmente positivamente relacionada ao tamanho do corpo. Os organismos maiores do solo (minhocas, térmitas e formigas), devido ao seu tamanho corporal influenciam as propriedades físicas do solo, e a biota menor (ácaros e principalmente microrganismos), atinge principalmente, a decomposição da serapilheira e as reações químicas que ocorrem nesses ambientes. Esses animais desempenham várias tarefas tanto nos ecossistemas naturais como nos

agricultáveis. A combinação dos efeitos químicos, físicos e biológicos dos animais do solo nas propriedades e em seus processos de preferência alimentar, como resíduos de raízes, deterioração de raízes e brotos, também podem influenciar significativamente o crescimento das plantas, ambos positivamente ou negativamente (BROWN et al., 2006).

Além de ser classificada de acordo com seu tamanho corporal, a fauna do solo também pode ser classificada de acordo com sua funcionalidade. Essa classificação pode ser dividida em saprófagos, micrófagos, predadores, insetos sociais e fitófagos.

Os saprófagos alimentam-se diretamente do material em decomposição, fragmentando-os. O seu hábito alimentar libera nutrientes em forma disponível às plantas.

Os micrófagos, são geralmente microartrópodes responsáveis pela regulação da população microbiana, pois se alimentam de microrganismos.

Os predadores alimentam-se de outros organismos vivos. A predação resulta em efeitos negativos no crescimento e sobrevivência de uma população que sofre predação e em efeito positivo ou benéfico na outra que executa a predação.

Os insetos sociais se caracterizam por apresentarem organização social, como é o caso das formigas (ordem Hymenoptera, família Formicidae) e dos cupins (ordem Isoptera). 


\section{A fauna do solo como indicadora da qualidade do solo}

A qualidade do solo é definida como a capacidade deste em funcionar dentro do ecossistema visando sustentar a produtividade biológica, manter a qualidade ambiental e promover a saúde das plantas e animais, sendo avaliada pelo uso de indicadores físicos, químicos e biológicos. O critério para o uso de um parâmetro como indicador do solo é a sua capacidade de interferir nos processos ecológicos; integrar as propriedades físicas, químicas e biológicas; além de ser facilmente utilizável por especialistas, técnicos e agricultores (ZATORRE, 2008).

As tentativas de uso de atividades biológicas como indicadores de avaliação de propriedades do solo não são recentes e a maioria dos estudos foi abandonada ainda no final da década de 1950, devido a problemas metodológicos, à concepção teórica inadequada e, conseqüentemente, a pouca sensibilidade para prever ou detectar mudanças no sistema. No final do século XX, esta abordagem retornou com muita intensidade, amparada nos avanços metodológicos (SILVEIRA et al., 2009).

Estudos da avaliação de atributos químicos, físicos e biológicos na qualidade do solo são essenciais no entendimento da funcionalidade e sustentabilidade de solos em diferentes condições de uso. O grande desafio dos estudos sobre sustentabilidade é com relação ao desenvolvimento de métodos para avaliação da qualidade do solo e do ambiente sob a interferência do homem. Há, atualmente, um esforço multidisciplinar, tentando quantificar diferentes atributos que estão relacionados com a sustentabilidade, traduzindo-os na forma de indicadores de qualidade do solo (MELLONI et al., 2008).

A fauna do solo pode ser utilizada para avaliar o status da sustentabilidade de um agroecossistema de produção, de recuperação de uma área degradada, ou de um sistema natural que foi modificado por ações antrópicas. De acordo com Lima et al. (2007), a estreita relação entre a fauna edáfica e a qualidade ambiental do solo, demonstra a importância desses organismos como indicadores do equilíbrio de funcionamento do sistema.

Os organismos têm papel importante na identificação da qualidade do solo, por atuarem nos processos de transformação da matéria orgânica, ciclagem de nutrientes e interações de troca com a maioria das espécies de plantas, especialmente as de importância agrícola. Dentre os organismos da fauna do solo, existem aqueles que influenciam direta ou indiretamente a disponibilidade de recursos para outros organismos, através da modificação do ambiente físico e químico (ZATORRE, 2008).

A maioria dos indivíduos pertencentes à meso e macrofauna melhoram a qualidade do solo, especialmente a mobilização de nutrientes, através de enzimas, e o melhoramento da estrutura, através da ativação da microvida. Melhoram a física do solo, removendo-o e cavando-o. A Galeria construída pelos animais de solo, como larvas de inseto, insetos, minhocas, besouros e outros servem à penetração das raízes, à infiltração de água e à circulação do ar. As enzimas excretadas por animais do solo estimulam o crescimento de plantas de cultura (FREITAS; BARRETO, 2008).

O húmus produzido pela macrofauna de minhocas melhora a Capacidade de Troca Catiônica (CTC) do solo. A meso e macrofauna fragmentam o material vegetal depositado sobre o solo, predigerindo-o, de modo que possa ser prontamente decomposto pelos microrganismos do solo.

Por possuírem muitas características benéficas ao solo, a macrofauna pode ser considerada uma boa indicadora da qualidade e da sustentabilidade do uso do solo, pois ela está envolvida em diversas funções e processos do solo, mostrando-se sensível às perturbações no ambiente, além de ser fácil e econômica de medir, auxiliando na tomada de decisões para um manejo ecológico mais sustentável do solo. Sua relação com as diferentes variáveis químicas e microbiológicas do solo, aliada à quantidade de cobertura vegetal (serapilheira) é uma excelente ferramenta para avaliação e monitoramento da qualidade ambiental (BARETTA, 2007).

Nucleus, v.9, n.2, out.2012 
O desenvolvimento de um sistema sustentável exige a utilização de indicadores mensuráveis, capazes de atestar o estado geral da qualidade do solo e com aplicabilidade em qualquer situação. Dentre estes, os que mais dependem de ações de pesquisa para serem desenvolvidos são aqueles baseados na diversidade e atividade da biota do solo. Neste contexto, a fauna do solo pode ser então, considerada como um bioindicador da qualidade do solo, auxiliando na orientação das mudanças na produtividade e no manejo conservacionista (MOÇO, 2006).

\section{Relação da qualidade do solo e da serapilheira com a fauna edáfica}

Os fragmentos orgânicos advindos dos componentes senescentes da parte aérea das plantas, ao caírem sobre o solo, formam uma camada denominada de serapilheira ou matéria orgânica, que compreende folhas, caules, flores e frutos, bem como restos de animais e material fecal, em diferentes estágios de decomposição. A vegetação é um fator determinante da variabilidade horizontal da serapilheira, pois quanto mais diversa for a comunidade vegetal, mais heterogênea será a serapilheira em pontos adjacentes. A heterogeneidade da serapilheira pode atuar aumentando a diversidade das comunidades da fauna do solo devido a um maior número de nichos a serem colonizados. Em ecossistemas onde a serapilheira depositada apresenta características diferenciadas, tais como baixa concentração de nutrientes e altos teores de lignina e de polifenóis totais, entre outros, ocorre uma diminuição das comunidades de invertebrados do solo (MOÇO, 2006).

A fauna do solo é a comunidade de invertebrados mais associada ao compartimento serapilheirasolo. Da sua associação com microrganismos, resulta uma participação nos processos de decomposição e ciclagem de nutrientes, assim como a modificação de propriedades químicas e físicas desse compartimento, devido à movimentação desses organismos no perfil do solo. O conjunto serapilheira-solo não representa somente fonte de carbono e energia para os organismos do solo, mas também o habitat onde todas as ações do organismo ocorrem, garantindo a sua sobrevivência e reprodução. A serapilheira é a porção mais dinâmica desse conjunto e, possivelmente, a mais variável (CORREIA; ANDRADE, 1999).

A vegetação é um fator determinante da variabilidade horizontal da serapilheira, pois quanto mais diversa for a comunidade vegetal, mais heterogênea será a serapilheira em pontos adjacentes (CORREIA; ANDRADE, 1999). Já que a camada de serapilheira é formada através da deposição sobre o solo de componentes advindos da parte aérea das plantas, como: folhas, caules, flores e frutos, que no solo se misturam aos restos de animais em diferentes estágios de decomposição.

Moço (2006) comprova a importância da serapilheira, pois ao estudar a distribuição da meso e macrofauna do solo e serapilheira com o tempo de amostragem em cinco diferentes agrossistemas de cacau no sul da Bahia, concluiu que o desenvolvimento de uma camada de serapilheira resultou em maior abundância e diversidade de fauna do solo, ou seja, a presença de mais fonte de nutrientes para a fauna contida na serapilheira, garantiu uma maior abundância e diversidade da fauna edáfica.

A qualidade da serapilheira, usualmente, é expressa em função do seu grau de lignificação, teores de nutrientes, compostos orgânicos solúveis, da presença de moléculas orgânicas com efeitos alelopáticos, assim como substâncias estimuladoras biologicamente significativas. Esta qualidade pode ser avaliada pela palatabilidade que afeta diretamente seu consumo pela fauna. A alguns polifenóis é atribuído o sabor amargo do material vegetal e assim são considerados fatores antinutricionais, afetando diretamente a preferência de consumo pela fauna. (MOÇO, 2006).

Os invertebrados do solo possuem uma baixa capacidade de digestão enzimática e se alimentam de proteínas, lipídeos e glicídeos simples. O processo de alimentação da fauna do solo é beneficiado pela atuação de microrganismos sobre a matéria orgânica, seja pela ingestão simultânea, onde a fauna se 
beneficia da degradação promovida pelos microrganismos e também do tecido destes, ou em interações mutualistas, onde os organismos vivem em simbiose. Os microrganismos são beneficiados pela atividade da fauna que contribui para a dispersão de populações microbianas e intensificam o processo de decomposição através da fragmentação dos resíduos vegetais. Esse processo otimiza a utilização das frações mais lábeis da matéria orgânica e favorece o acúmulo de matéria orgânica recalcitrante em coprólitos e agregados (SILVA, 2006).

Dentro deste contexto, Bianchi et al. (2006) avaliaram a taxa de consumo pela fauna do solo, de serapilheira oriunda de diferentes espécies arbóreas observando que Mimosa caesalpinifolia (Sabiá) e Pseudosamanea guachapelle (Guachapele) foram as espécies mais consumidas, chegando a um consumo três vezes maior do que um segundo grupo, formado por Erythrina poepigiana (Mulungu) e Acacia auriculiformis (Acácia). Por outro lado, as serapilheiras menos consumidas foram das espécies Saman, Ingá e Jamelão. No caso das duas últimas e da Acácia, a elevada relação $\mathrm{C} / \mathrm{N}$ parece ter sido a principal responsável pela inibição do consumo. No entanto, para Saman e Mulungu, este não parece ter sido o principal efeito de inibição, pois as mesmas apresentaram baixos valores da relação $\mathrm{C} / \mathrm{N}$. Como os valores de polifenóis e $\mathrm{N}$ são referentes à análise do material ainda verde, apesar do considerável teor de polifenóis apresentados por Sabiá e Guachapele, esses podem ter sido lixiviados durante o período em que as folhas permaneceram no solo antes de serem coletadas, justificando o alto consumo pelos organismos do grupo diplópoda.

Da mesma forma, Bianchi e Correia (2007) avaliaram o consumo de material verde e da serapilheira de diferentes espécies vegetais exercida pelos diplópodas. Concluindo que o material mais consumido foi o de Mimosa caesalpinifolia (sabiá) tanto em material verde como na forma de serapilheira, destacando a serapilheira, que teve um valor de consumo de aproximadamente três vezes o do material verde.

Os organismos da fauna do solo são afetados por fatores como temperatura, umidade, textura, porosidade, entre outros. Por exemplo, alta umidade e baixa temperatura do solo devem ser mantidas, de modo que, se a superfície do solo ressecar prejudicará a fauna edáfica. A textura e a porosidade do solo são características altamente importantes determinando, em grande parte, a disponibilidade de nutrientes para os animais do solo e as plantas (MOÇO, 2006).

Freitas e Barreto (2008) relacionaram a qualidade do solo com a densidade de fauna, riqueza de espécies e a distribuição das comunidades edáficas nas coberturas vegetais, Mata e monocultura de café. Encontrando densidade de fauna e riqueza de espécies em maior número na Mata, sugerindo que esta está sujeita a uma maior ciclagem e reposição de nutrientes e, portanto a modificações significativas de ordem funcional e estrutural. A maior riqueza de espécies encontrada na Mata também pode ser explicada pelo fato da mesma possuir melhores condições de temperatura, umidade e disponibilidade de alimentos, favoráveis à manutenção da maioria dos organismos bioindicadores.

Cole et al. (2006) ao estudarem os efeitos de perturbações antropogênicas na biota do solo. Concluíram que, a adição de nutrientes teve efeitos diretos e indiretos sobre a abundância e estrutura da comunidade da fauna edáfica. Como por exemplo, a adição de calcário afetou a comunidade e a estrutura do grupo das minhocas. Em contraste, os efeitos indiretos de adições de nutrientes no solo foram exercidos sobre a comunidade de microartrópodes através da alteração da estrutura e produtividade da comunidade vegetal, que por sua vez afetou a comunidade da fauna do solo. A adição de nutrientes aumentou a produtividade da planta, proporcionando maiores recursos para a fauna do solo o que resultou em aumento da biomassa para alguns grupos.

Estes estudos demonstram a importância e a inter-relação da qualidade do solo e da serapilheira, que servirá de abrigo e alimento para a fauna do solo respectivamente. 


\section{Análise multivariada no estudo de bioindicadores da qualidade do solo e da serapilheira}

A diversidade da fauna do solo tem importância tanto no aspecto ecológico como para a qualidade do solo. Entretanto, até o momento, não existe um bioindicador do solo que permite avaliar a sua qualidade de maneira rápida, econômica e eficiente. Como o número de indicadores é enorme, a aplicação de índices de qualidade e de análise estatística multivariada pode ajudar o pesquisador na interpretação dos resultados (BARETTA, 2007).

Os organismos do solo e suas comunidades estão continuamente mudando e se adaptando às alterações ambientais. A dinâmica natural desse grupo faz deles indicadores potencialmente sensíveis para se avaliar as mudanças no solo, resultantes de diferentes práticas e sistemas de manejo. O desafio é identificar parâmetros do solo mensuráveis que possam ser usados para avaliar as práticas de manejo do solo para um dado local. Como o número possível de indicadores biológicos é amplo, a análise multivariada surge como importante alternativa em estudos dessa natureza (FACCI, 2008).

Embora os estudos que envolvem os atributos biológicos e químicos do solo tenham aumentado muito nos últimos anos, devido ao grande interesse em elucidar os processos que ocorrem nos agrossistemas, de acordo com Maluche-Baretta et al. (2006), o modelo de análise estatística univariada convencional torna-se menos sensível em sistemas biológicos, em razão das particularidades de cada manejo tratando somente uma das diversas variáveis a cada tempo, e não considera o efeito conjunto dos inúmeros atributos químicos e biológicos avaliados (MARIANI et al., 2006).

A idéia de utilizar técnicas estatísticas que permitam a ordenação de amostras em função de uma série de fatores ambientais simultaneamente permite uma análise conjunta dos fatores ambientais para verificação de suas correlações com diferentes ecossistemas ou usos do solo (MELLONI et al., 2008). Os métodos de análise multivariada são importantes ferramentas no estudo de indicadores de qualidade do solo, pois estabelecem relações entre os atributos químicos e biológicos (BARETTA; MALUCHEBARETTA; CARDOSO, 2008).

Como exemplo de análises multivariadas amplamente utilizadas no estudo de atributos indicadores da qualidade do solo estão as análises multivariadas canônicas, como a análise canônica discriminante (ACD) e a análise de correlação canônica (ACC). A análise canônica discriminante (ACD) é definida por Baretta et al. (2005), como uma ferramenta estatística, que permite estabelecer não só a identificação de diferenças entre tratamentos, mas também qual é o atributo que mais contribui para a separação entre as áreas estudadas, permitindo, assim, a eliminação de tempo e dinheiro gastos com aqueles atributos do solo que expressam baixa resposta.

Maluche-Baretta et al. (2006) identificaram diferenças entre os sistemas de produção convencional e orgânico de maçãs quanto aos atributos microbiológicos do solo, por meio de modelos multivariados que utilizam a análise canônica discriminante (ACD) e análise de correlação canônica (ACC). Chegando a conclusão que métodos de análise multivariada são importantes no estudo das alterações de atributos do solo, pois existiu alta correlação canônica entre atributos biológicos e químicos do solo nas áreas estudadas pelos autores.

Coimbra, et al. (2007) estudaram uma alternativa para a avaliação da fauna do solo por meio de alguns indicadores biológicos, utilizando análise multivariada. Mostrando que a análise discriminante

canônica demonstra quais os organismos de maior peso canônico que auxiliam na diferenciação das áreas nas diferentes coletas.

Da mesma forma, Baretta; Maluche-Baretta; Cardoso (2008) estudando atributos microbianos e químicos do solo, por meio de métodos multivariados, como a análise canônica discriminante (ACD) e a análise de correlação canônica (ACC) em florestas com araucária naturais e reflorestadas, demonstraram 
que os dois métodos são importantes ferramentas no estudo de indicadores de qualidade do solo, pois estabelecem relações entre os atributos químicos e microbianos e também que a análise canônica discriminante (ACD) mostrou que a contribuição de cada atributo microbiano para a separação das áreas sofre variação ao longo do tempo.

Outro exemplo de análise multivariada é a análise de correspondência, que separa os tratamentos, demonstra associações entre os grupos da fauna edáfica e os sistemas de manejo do solo. A partir da identificação destes grupos de organismos, podem se desenvolver estudos complementares, com maior detalhamento taxonômico, para se verificar a contribuição desses animais, em termos de serviços ecológicos no solo, o que ampliaria sua utilização como bioindicadores da qualidade do solo (BARETTA, et al., 2006).

Baretta, et al., (2006) avaliaram o efeito de sistemas de preparo e cultivo do solo sobre grupos da fauna edáfica, utilizando análise de correspondência, indicando que a permanência, o estabelecimento e os grupos da fauna edáfica presentes são sensíveis às operações de preparo e cultivo do solo. Evidenciando que a fauna do solo pode ser utilizada como bioindicador das alterações advindas do manejo do solo, especialmente pelo emprego de ferramentas estatísticas multivariadas.

Melloni et al. (2008) utilizando ferramentas de agrupamento e análise multivariada, estudaram efeitos de coberturas florestais e de pastagem na qualidade química, física e biológica de solos no Sul de Minas Gerais. Os autores observaram que quando as variáveis ambientais (atributos físicos, químicos e biológicos) do solo são analisadas em conjunto e correlacionadas com diferentes ecossistemas, a visualização e a ordem de influência dessas variáveis são bem mais claras. Através da análise multivariada, esses autores observaram a total dissimilaridade da pastagem com relação aos demais ecossistemas. Sendo os atributos químicos ligados à fertilidade $\left(\mathrm{P}, \mathrm{K}, \mathrm{Ca}^{2+}, \mathrm{Mg}^{2+}, \mathrm{pH}, \mathrm{SB}\right.$ e V\%), além da densidade do solo, os atributos responsáveis por promoverem a total separação da pastagem dos outros ecossistemas.

Dentro do contexto das análises multivariadas, surge a necessidade de se utilizar a análise de trilha sempre que se deseja estudar os efeitos diretos e ou indiretos de uma variável sobre a outra, pois os coeficientes de correlação, apesar de serem de grande utilidade na quantificação da magnitude e direção das influências de fatores na determinação de caracteres complexos, não dão a exata importância relativa dos efeitos diretos e indiretos destes fatores. Estudos sobre o desdobramento do coeficiente de correlação são feitos pela análise de trilha, desenvolvida por Wrigth (1923) e pormenorizada por Li (1975).

A análise de trilha (ou path analysis) consiste no desdobramento de coeficientes de correlação entre a variável principal e cada variável explicativa, em efeitos diretos e indiretos destas em relação àquela. Os efeitos diretos das variáveis independentes sobre a variável principal são os coeficientes do modelo de regressão padronizada. O efeito indireto de uma variável independente sobre a principal, via outras variáveis independentes, são aqueles proporcionados pelas correlações entre essas variáveis independentes (SOUZA et al., 1999).

Esta análise possibilita avaliar os efeitos diretos das variáveis independentes (explicativas) sobre uma variável dependente (básica). E permite também verificar os efeitos indiretos entre as variáveis explicativas sobre a variável básica. A análise de trilha também mostra o efeito total de um atributo sobre outro. Este efeito total considera a junção dos efeitos diretos e indiretos, desta forma quando um atributo (x) exerce efeito total sobre o atributo (y), significa que o atributo $(\mathrm{x})$ influencia o atributo (y) mais que simplesmente e isoladamente de forma direta ou de forma indireta, mas de forma total, completa, levando em consideração uma junção dos efeitos diretos e indiretos.

Alguns estudos relatam a inter-relação dos atributos químicos do solo e da serapilheira com a comunidade da fauna edáfica utilizando análise de trilha, esta análise é uma ótima ferramenta de 
inferência das relações diretas e indiretas, com um potencial de aplicação elevado, que ainda está sendo descoberto para estudos da biologia do solo.

Trabalhos como os de Moço et al. (2010); Towns et al. (2009); Lussenhop, (1996), tentam elucidar algumas questões das relaçoes dos atributos do solo e da serapilheira com a fauna edáfica utilizando análise de trilha.

Moço et al. (2010) ao estudarem fauna edáfica em agrossistemas de cacau no Sul da Bahia, utilizando análise de trilha, relataram que atributos como $\mathrm{pH}$, soma de bases e argila foram os que mais influenciaram a densidade da fauna edáfica nos solos das áreas estudadas.

Towns et al. (2009) também utilizando análise de trilha, encontraram efeito significativo do pH sobre alguns grupos de fauna principalmente o grupo Psocoptera. Outros trabalhos também relatam a importância do $\mathrm{pH}$ do solo como um atributo muito importante para a estruturação de comunidades do grupo Collembola.

Lussenhop (1996) encontrou relação direta dos teores de nitrogênio e fósforo do solo com a densidade do grupo Collembola utilizando análise de trilha, o primeiro nutriente influenciando de forma negativa e o segundo de forma positiva. O grupo Collembola é composto por indivíduos extremamente sensíveis, o que permite que manifestem rapidamente as conseqüências às variações ambientais em suas populações, por isto é um dos grupos da fauna edáfica que tem merecido destaque como indicador biológico. No entanto, o conhecimento das interações ecológicas no solo e da diversidade de colêmbolos edáficos na Mata Atlântica ainda é bastante escasso (BARETTA; MALUCHE-BARETTA; CARDOSO, 2008) se tornando muito importante de ser estudado, pois a diversidade da estrutura da cobertura vegetal influencia a variabilidade da distribuição de comunidades de colêmbolos edáficos.

\section{CONCLUSÃO}

A comunidade da fauna do solo e da serapilheira mostra-se influenciada pelos atributos que constituem o solo e a serapilheira de diferentes coberturas vegetais.

A composição da comunidade da fauna do solo parece ser regulada principalmente pelos teores de nitrogênio, fósforo e carbono e pelo $\mathrm{pH}$ do solo, enquanto a comunidade da fauna da serapilheira parece ser influenciada, sobretudo pelos teores de nitrogênio, carbono, lignina e polifenóis da serapilheira.

$\mathrm{O}$ estudo da inter-relação fauna versus atributos contribui para o entendimento de como atributos do solo e da serapilheira relacionados à nutrição, acidez, palatabilidade e microclima afetam a colonização, sobrevivência e dinâmica da comunidade da fauna sob diferentes coberturas vegetais.

Os grupos de fauna podem ser influenciados positivamente ou negativamente pelos atributos químicos da serapilheira e do solo.

A utilização de análises multivariadas demonstra ser uma ferramenta promissora para explicar a correlação entre diferentes variáveis que apresentam unidades diferentes no estudo de bioindicadores da qualidade do solo e suas influências com as características químicas e físicas do solo e da serapilheira.

\section{REFERÊNCIAS}

BARETTA, D.et al. Efeito do monocultivo de pinus e da queima do campo nativo em atributos biológicos do solo no planalto sul catarinense. Revista Brasileira de Ciência do Solo, v. 29, p. 715-724, 2005. 
BARETTA, D.et al. Efeito do cultivo do solo sobre a diversidade da fauna edáfica no planalto sul catarinense. Revista de Ciências Agroveterinárias, Lages, v.5, n.2, p. 108-117, 2006.

BARETTA, D. Fauna do solo e outros atributos edáficos como indicadores da qualidade ambiental em áreas com Araucaria angustifolia no Estado de São Paulo. 2007. 158 f. Tese (Doutorado) Universidade de São Paulo. Escola Superior de Agricultura Luiz de Queiroz. Piracicaba.

BARETTA, D.; MALUCHE BARETTA, C. R. D.; CARDOSO, E. J. B. N. Análise multivariada de atributos microbiológicos e químicos do solo em florestas com Araucaria angustifólia. Revista Brasileira de Ciência do Solo, v. 32, p. 2683-2691, 2008.

BIANCHI, M. O.et al. Avaliação da palatabilidade e da taxa de consumo de diferentes tipos de serrapilheira pela fauna do solo. In: CONGRESSO BRASILEIRO DE SISTEMAS AGROFLORESTAIS, 6. 2006. Anais... 2006.

BIANCHI, M. DE O.; CORREIA, M. E. F. Mensuração do consumo de material vegetal depositado sobre o solo por diplópodes. Seropédica, 2007. (Circular técnica ,20).

BROWN, G.G.et al. Biodiversity and function of soil animals in Brazilian agroforestry systems. In: GAMA-RODRIGUES, A.C.et al. (Eds.) Sistema Agroflorestais: Bases científicas para o desenvolvimento sustentável. Campos dos Goytacazes, RJ : Universidade Estadual do Norte Fluminense UENF, 2006. p. $217-242$.

COIMBRA, J. L. M.et al. Técnicas multivariadas aplicadas ao estudo da fauna do solo: contrastes multivariados e análise canônica discriminante. Revista Ceres, v. 54, n. 313, p. 270-276, 2007

COLE, L.et al. The abundance, richness and functional role of soil meso- and macrofauna in temperate grassland:A case study. Applied Soil Ecology, v.33, n. 186-198, 2006.

CORREIA, M.E.F.; ANDRADE, A.G. Formação de serapilheira e ciclagem de nutrientes. In: SANTOS, G. A., CAMARGO, F. A. O., (eds.) Fundamentos da matéria orgânica do solo: Ecossistemas tropicais e subtropicais. Porto Alegre: Genesis. p. 197-225, 1999.

CORREIA, M.E.F.; OLIVEIRA, L.C.M. Fauna de solo: aspectos gerais e metodológicos. Seropédica: Embrapa Agrobiologia. P. 46, 2000. (Embrapa Agrobiologia. Documento, 112).

DEVIDE, A.C.P.; CASTRO, C.M. Manejo do solo e a dinâmica da fauna edáfica. 2009. Disponível em: <http://www.infobibos.com/Artigos/2009_1/ManejoSolo/index.htm>. Acesso em: 3 set. 2009.

FACCI, L. D. Variáveis microbiológicas como indicadoras da qualidade do solo sob diferentes usos. 104f. 2008. Dissertação (Mestrado) Instituto Agronômico de Campinas.

FREITAS, A. C. S.; BARRETO, L. V. Qualidade biológica do solo em ecossistemas de mata nativa e monocultura do café. Goiânia: Instituto Construir e Conhecer; Enciclopédia Biosfera n.5, 2008.

LI, C. C. Path analysis - a primer. Boxwood: Pacific Grove, 346p. 1975.

LIMA, S. S.et al. Diversidade da macrofauna edáfica em agroflorestas de diferentes estádios sucessionais. Revista Brasileira de Agroecologia. v. 2, n. 2, p. 1029-1033, 2007.

LUSSENHOP, J. Collembola as mediators of microbial symbiont effects upon soybean. Soil Biol. Biochem. v.28. n.3, p. 363-369, 1996.

MALUCHE-BARETTA, C. R. D.; AMARANTE, C. V. T.; FILHO, O. K. Análise multivariada de atributos do solo em sistemas convencional e orgânico de produção de maçãs Pesq. agropec. bras., Brasília, v.41, n.10, p.1531-1539, 2006 
MARIANI, L.; CHANG, S.X.; KABZEMS, R. Effects of tree harvesting, forest floor removal, and compaction on soil microbial biomass, microbial respiration, and $\mathrm{N}$ availability in a boreal aspen forest in British Columbia. Soil Biol. Biochem., v. 38, p.1734-1744, 2006.

MELLONI, R.et al. Avaliação da qualidade de solos sob diferentes coberturas florestais e de pastagem no sul de Minas Gerais. Revista Brasileira de Ciência do Solo, v. 32, p. 2461-2470, 2008.

MOÇO, M. K. S.et al. Caracterização da fauna edáfica em diferentes coberturas vegetais na região Norte Fluminense. Revista brasileira de ciência do solo. v. 29, p.555- 564, 2005.

MOÇO, M. K.DA S. Fauna do solo em diferentes agrossistemas de cacau no sul da Bahia. 93f. 2006. Dissertação (Mestrado). Universidade Estadual Do Norte Fluminense Darcy Ribeiro. Campos dos Goytacazes-RJ

MOÇO, M. K. S. et al. Relationships between invertebrate communities, litter quality and soil attributes under different cacao agroforestry systems in the south of Bahia, Brazil. Applied Soil Ecology, v.46, p.347-354, 2010.

SILVA, M. S. C. DA. Indicadores de qualidade do solo em Sistemas Agroflorestais em Paraty, RJ. 54f. 2006. Dissertação (Mestrado). Universidade Federal Rural do Rio de Janeiro. Seropédica.

SILVEIRA, A. O.et al. Processos biológicos como indicadores de qualidade ambiental. Boletim Informativo da Sociedade Brasileira de Ciência do Solo. jan- abr. p. 15. 2009.

SOUZA, J. O. JR.et al. Produtividade do cacaueiro em função de características do solo. I. Características químicas. Revista Brasileira de Ciência do Solo. v.23, p. 863- 872, 1999.

TOWNS, D. R.et al. Predation of seabirds by invasive rats: multiple indirect consequences for invertebrate communities. Oikos, v. 118, p. 420-430, 2009.

WRIGHT, S. Theory of path coefficients. Genetics, New York, v.8, p.239-285, 1923.

ZATORRE, N.P. Atributos biológicos do solo como indicadores de qualidade do solo. Gaia Scientia, v. 2, n. 1, p. $9-13,2008$. 
Nucleus, v.9, n.2, out.2012 